\title{
A parametrization of the solutions of the Hamiltonian system for stabilizable pairs*
}

\author{
Giovanni Marro Lorenzo Ntogramatzidis \\ Dipartimento di Elettronica, Informatica e Sistemistica \\ Università di Bologna, viale Risorgimento, 2 - 40136 Bologna, Italy \\ tel: +390512093046 - fax: +390512093073 \\ \{gmarro,lntogramatzidis\}@deis.unibo.it
}

March 2, 2005

\begin{abstract}
In this note, a simple method is proposed for the solution of the finite-horizon LQ problem, which does not require the integration of the Riccati differential equation. Precisely, the problem is tackled by parametrizing the set of trajectories solving the Hamiltonian system in finite terms, under the assumption of stabilizability of the underlying system. In this way, it is possible to determine closed-form expressions for the state and control functions, as well as for the optimal cost in terms of the assigned state at the end-points.
\end{abstract}

Keywords: finite-horizon LQ, Hamiltonian system, algebraic Riccati equations, boundary conditions, optimal cost.

${ }^{*}$ Partially supported by the ministry of higher education of Italy (MIUR), under project Identification and Control of Industrial Systems. 


\section{Introduction}

This paper addresses the finite-horizon LQ regulator. As it is well-known, this problem is usually tackled in the literature by resorting to the solution of a suitable matrix differential Riccati equation with assigned terminal condition, see e.g. Anderson et al. (1989), Kwakernaak et al. (1972), Lewis et al. (1995). However, such equation is typically difficult to solve, unless approximate algorithms based on finite-difference methods are employed. In the recent literature, a new technique for the solution of this problem has been presented for controllable systems, Ferrante et al. (2003), which relies on a simple formula parametrizing the solutions of the associated Hamiltonian system in terms of the stabilizing and anti-stabilizing solutions of the infinite-horizon algebraic Riccati equation.

In this paper, we extend the material presented in Ferrante et al. (2003) to the case when the underlying system is stabilizable, thus weakening the controllability assumption. In this case, in fact, the anti-stabilizing solution of the associated ARE does not exist, and a new formula is established in order to obtain a complete parametrization of the trajectories satisfying the Hamiltonian system involving the sole stabilizing solution. The advantages of the present approach are both theoretical and practical. In fact, from the one side this method also applies to non-standard LQ problems (like the fixed end-point LQ); from the other side, the parametrization of the solutions of the Hamiltonian system proposed herein is computationally attractive, since it involves:

- the solution of two algebraic equations, i.e., an ARE and a Lyapunov equation, which are easily computable through standard and currently available software routines (see e.g. the MATLAB ${ }^{\circledR}$ functions care.m and lyap.m);

- two exponentials of strictly stable matrices. This fact ensures that the present solution is robust even for large time horizons.

Finally a closed-form expression of the optimal cost is also presented, as a quadratic form in the assigned state(s).

\section{Statement of the problem}

Consider the LTI continuous-time system described by

$$
\begin{aligned}
& \dot{x}(t)=A x(t)+B u(t), \quad x(0)=x_{0} \in \mathbb{R}^{n}, \\
& y(t)=C x(t)+D u(t),
\end{aligned}
$$

where, for all $t \geq 0, x(t) \in \mathbb{R}^{n}$ is the state, $u(t) \in \mathbb{R}^{m}$ is the input, $y(t) \in \mathbb{R}^{p}$ is the output. Let $A$, $B, C$ and $D$ be real, constant matrices of suitable dimensions. Let $D$ be full column rank.

The first optimal control problem considered in this note is the traditional LQ regulator, in which the terminal state is weighted quadratically in the functional to be minimized.

Problem 1 Let $T>0$. Given a terminal state penalty matrix $P_{T}=P_{T}^{\top} \geq 0$, find a measurable $u(t), t \in[0, T)$, and an absolutely continuous $x(t), t \in[0, T]$, minimizing the quadratic cost

$$
J_{1}(x, u)=\int_{0}^{T} y^{\top}(t) y(t) d t+x^{\top}(T) P_{T} x(T)
$$


under the constraints (1)-(2).

The second optimization problem dealt with is the fixed end-point LQ, in which the terminal state is assigned.

Problem 2 Let $T>0$. Given a terminal state $x_{T} \in \mathbb{R}^{n}$ reachable from $x_{0}$, find a measurable $u(t), t \in[0, T)$, and an absolutely continuous $x(t), t \in[0, T]$, minimizing the quadratic cost

$$
J_{2}(x, u)=\int_{0}^{T} y^{\top}(t) y(t) d t
$$

under the constraints (1)-(2) and $x(T)=x_{T}$.

Remark 1 The case when the terminal state is assigned and the initial state is weighted in the performance index is easily obtained as the symmetric of Problem 1.

In this note, we make the following standing assumptions:

(A1) the pair $(A, B)$ is stabilizable;

(A2) the quadruple $(A, B, C, D)$ has no invariant zeros on the imaginary axis.

\section{Hamiltonian state-costate trajectories}

Let $Q:=C^{\top} C, R:=D^{\top} D$ and $S:=C^{\top} D$. It is well-known - see e.g. Lewis, Syrmos (1995) that a necessary set of conditions for optimality are expressed by the Hamiltonian system

$$
\left[\begin{array}{l}
\dot{x}(t) \\
\dot{\lambda}(t)
\end{array}\right]=H\left[\begin{array}{l}
x(t) \\
\lambda(t)
\end{array}\right], \quad \text { where } \quad H:=\left[\begin{array}{cc}
A-B R^{-1} S^{\top} & -B R^{-1} B^{\top} \\
-Q+S R^{-1} S^{\top} & -A^{\top}+S R^{-1} B^{\top}
\end{array}\right]
$$

is called the Hamiltonian matrix and $\lambda(t) \in \mathbb{R}^{n}, t \in[0, T]$ is the costate, along with the so-called stationarity condition

$$
u(t)=-R^{-1}\left(S^{\top} x(t)+B^{\top} \lambda(t)\right) .
$$

Recall that if assumptions (A1)-(A2) hold, the matrix $H$ has no eigenvalues on the imaginary axis - see Zhou et al. (1996: Theorem 13.7, Lemma 13.9 and Corollary 13.10) - and the ARE

$$
P A+A^{\top} P-(S+P B) R^{-1}\left(S^{\top}+B^{\top} P\right)+Q=0
$$

admits a maximal solution $P_{+}=P_{+}^{\top} \geq 0$, which is stabilizing, i.e., all the eigenvalues of the closed-loop matrix $A_{+}:=A-B K_{+}$, where $K_{+}:=R^{-1}\left(S^{\top}+B^{\top} P_{+}\right)$, have strictly negative real part, Molinari (1977). Moreover, under assumption (A1) the Lyapunov equation

$$
A_{+} W+W A_{+}^{\top}+B R^{-1} B^{\top}=0
$$

has a unique solution $W \in \mathbb{R}^{n \times n}$, which is symmetric positive semidefinite: Ionescu et al. (1999: Theorem 1.5.5).

The following result consists of a closed-form parametrization of all the solutions of the Hamiltonian system. 
Theorem 1 The set of trajectories solving the Hamiltonian system (5) is parametrized in $p, q \in \mathbb{R}^{n}$ as

$$
\left[\begin{array}{l}
x(t) \\
\lambda(t)
\end{array}\right]=\left[\begin{array}{c}
I \\
P_{+}
\end{array}\right] e^{A_{+} t} p+\left[\begin{array}{c}
W \\
P_{+} W-I
\end{array}\right] e^{-A_{+}^{\top}(t-T)} q .
$$

Proof: Let $J_{+}$and $J_{-}$denote the first and the second $2 n \times n$ matrices in the right-hand side of (9), respectively. By taking (7) and (8) into account, after a simple algebraic computation it is found that $H J_{+}=J_{+} A_{+}$and $H J_{-}=-J_{-} A_{+}^{\top}$. Now, by substitution it is easily seen that the set of trajectories (9) satisfies the Hamiltonian system (5) for all $p$ and $q$.

Conversely, we prove that all the solutions of (5) can be expressed by means of (9) for suitable values of $p$ and $q$. First, note that the order of the linear differential equation (5) is $2 n$, hence (5) admits $2 n$ linearly independent solutions. It will be shown that we may select $2 n$ linearly independent solutions from (9). To this aim, note that from $H J_{+}=J_{+} A_{+}$and $H J_{-}=-J_{-} A_{+}^{\top}$ it follows that $i m J_{+}$and $i m J_{-}$are $H$-invariant subspaces, and that the eigenvalues of $H$ restricted to $i m J_{+}$and $i m J_{-}$are all stable and anti-stable, respectively. As a result, the intersection $i m J_{+} \cap i m J_{-}$is zero. Hence, for any given pair $p, q \in \mathbb{R}^{n}$ the two trajectories $J_{+} e^{A_{+} t} p$ and $J_{-} e^{A_{+}^{\top}\left({ }^{(t-T)}\right.} q$ are linearly independent. Therefore, the dimension of the linear space of trajectories in (9) is given by the sum of the dimensions $n_{1}$ and $n_{2}$ of the subspaces of trajectories of (9) corresponding to $p=0$ and to $q=0$, respectively. Now we show that $J_{+}$ and $J_{-}$are both full column rank: this is obvious for $J_{+}$. Concerning $J_{-}$, let $v$ be a vector of its null-space. Then $W v=0$ and $\left(P_{+} W-I_{n}\right) v=v=0$. Hence, since both $J_{+}$and $J_{-}$are full column rank and the matrix exponentials are non-singular for all $t \in[0, T]$, it follows that $n_{1}=n$ and $n_{2}=n$, respectively. We may conclude that (9) admits $2 n$ linearly independent solutions.

Remark 2 The expressions of the state-costate functions satisfying the Hamiltonian system are given in terms of the matrix exponentials $\exp \left[A_{+} t\right]$ and $\exp \left[A_{+}^{\top}(T-t)\right]$. Hence, the optimal solution involves exponentials of strictly stable matrices in the overall time interval $[0, T]$, thus ensuring that its computation is numerically robust even for large time horizons. Furthermore, the matrices $P_{+}, A_{+}$and $W$ may be computed by standard and reliable algorithms available in any control package (see e.g. the MATLAB ${ }^{\circledR}$ routines care.m and lyap.m).

\section{Boundary conditions and optimal control}

As already observed, the Hamiltonian system is a set of necessary equations for optimality. Hence, the optimal solution for both Problems 1 and 2 has the form of (9), for suitable values of the parameters $p$ and $q$. In order to obtain a set of conditions which are also sufficient, we have to add the suitable boundary conditions to the Hamiltonian system (5) and to the stationarity condition (6), see Mangasarian (1966). In other words, the correct boundary conditions have to be imposed on (9), so as to obtain the optimal state and costate trajectories.

\section{Boundary conditions for Problem 1}

Let $F:=e^{A_{+} T}$ and $\pi:=\left[\begin{array}{ll}p^{\top} & q^{\top}\end{array}\right]^{\top}$. If we impose the boundary equations $x(0)=x_{0}$ and 
$\lambda(T)=P_{T} x(T)$, Lewis et al. (1995), to the trajectories in (9), we obtain two equations in $p$ and $q$ that can be written in the compact form

$$
N_{1} \pi=w_{1}, \quad \text { with } \quad N_{1}:=\left[\begin{array}{cc}
I & W F \\
\left(P_{+}-P_{T}\right) F & \left(P_{+}-P_{T}\right) W-I
\end{array}\right], \quad w_{1}:=\left[\begin{array}{c}
x_{0} \\
0
\end{array}\right] .
$$

It is easily shown by contradiction that $N_{1}$ is non-singular. Suppose that two vectors $p, q \in \mathbb{R}^{n}$ exist such that the non-null vector $\pi$ belongs to the null-space of $N_{1}$. The corresponding statetrajectory following from (9) is such that $x(0)=0$. This trajectory is identically zero. In fact, the cost associated with the identically zero state trajectory and control law is zero. It follows that the cost associated with this trajectory cannot be greater than zero. On the other hand, due to the injectivity of $D$, the realization $(A, B, C, D)$ is left-invertible, hence any non-null input function would give rise to a strictly positive value of the integral in (3), and the cost would not be zero, see Trentelman et al. (2001: p.163 and p.189). It follows that the input is zero, and so is the state function. However, non-null values of $p$ and $q$ are not compatible with an identically null state trajectory: in fact, the solutions of (5), represented by (9), are elements of a $2 n$-dimensional vector space which is isomorphic to $\mathbb{R}^{2 n}$, see proof of Theorem 1. If a vector $\pi \neq 0$ existed such that the corresponding state-costate trajectory is identically zero, the parametrization given by (9) would be isomorphic to a vector space whose dimension is smaller than $2 n$. It follows that the origin of the space of all solutions of the Hamiltonian system, which is the identically zero state-costate trajectory, is obtained uniquely by assuming $\pi=0$; hence the null-space of $N_{1}$ is zero.

\section{Boundary conditions for Problem 2}

If we impose the boundary equations $x(0)=x_{0}$ and $x(T)=x_{T}$ to the trajectories in (5), we obtain two equations in $p$ and $q$ that can be written in the compact form

$$
N_{2} \pi=w_{2}, \quad \text { with } \pi:=\left[\begin{array}{c}
p \\
q
\end{array}\right], \quad N_{2}:=\left[\begin{array}{cc}
I & W F^{\top} \\
F & W
\end{array}\right], \quad w_{2}:=\left[\begin{array}{c}
x_{0} \\
x_{T}
\end{array}\right] .
$$

In this case, the problem admit solutions if and only if $x_{T}$ is reachable from the initial state $x_{0}$, Berkovitz (1964). If this is the case, the linear equation (11) admits solutions, i.e., $w_{2} \in i m N_{2}$. The set of parameters $\pi$ providing the optimal solution is parametrized in $k e r N_{2}$ as

$$
\pi=N_{2}^{+} w_{2}+v, \quad v \in \operatorname{ker} N_{2}
$$

However, the values of $p$ and $q$ satisfying the latter yield the same (optimal) state trajectory and control function, since the system is left-invertible.

\section{Derivation of the optimal control}

The solution of Problems 1 and 2 is derived as

$$
\left[\begin{array}{l}
x(t) \\
u(t)
\end{array}\right]=\left[\begin{array}{cc}
e^{A_{+} t} & W e^{A_{+}^{\top}(T-t)} \\
-K_{+} e^{A_{+} t} & -R^{-1}\left(S^{\top} W+B^{\top} P_{+} W-B^{\top}\right) e^{A_{+}^{\top}(T-t)}
\end{array}\right] \pi, \quad \pi \in \Pi,
$$

where $\Pi:=\left\{\pi=N_{1}^{-1} w_{1}\right\}$ in the case of Problem 1 and $\Pi:=\left\{\pi=N_{2}^{+} w_{2}+v: v \in \operatorname{ker} N_{2}\right\}$ in the case of Problem 2. 
The following theorem provides general expressions for the optimal value of the cost functions corresponding to the two optimal control problems described in Section 2, which can be expressed as a quadratic form in the assigned extreme states.

Theorem 2 Let $F:=e^{A_{+} T}$. Consider Problem 1. Let $N_{1}$ be defined in (10) and consider the partition $N_{1}^{-1}=\left[\begin{array}{ll}M_{1} & M_{2}\end{array}\right]$, where $M_{1}, M_{2} \in \mathbb{R}^{2 n \times n}$. The optimal cost is given by the following quadratic form in $x_{0}$ :

$$
J_{1}^{o}=x_{0}^{\top} M_{1}^{\top}\left[\begin{array}{ll}
\Psi_{1} & \Psi_{2} \\
\Psi_{2}^{\top} & \Psi_{3}
\end{array}\right] M_{1} x_{0},
$$

where $\Psi_{1}:=P_{+}-F^{\top}\left(P_{+}-P_{T}\right) F, \Psi_{2}:=P_{+} W F^{\top}-F^{\top}\left(P_{+}-P_{f}\right) W$ and $\Psi_{3}:=F^{\top} W\left(P_{+} W-\right.$ I) $F-W\left(\left(P_{+}-P_{T}\right) W-I\right)$. Consider Problem 2. The optimal cost is given by the following quadratic form in $x_{0}$ and $x_{T}$ :

$$
J_{2}^{o}=\left[\begin{array}{ll}
x_{0}^{\top} & x_{T}^{\top}
\end{array}\right]\left(N_{2}^{+}\right)^{\top}\left[\begin{array}{ll}
\Phi_{1} & \Phi_{2} \\
\Phi_{2}^{\top} & \Phi_{3}
\end{array}\right] N_{2}^{+}\left[\begin{array}{l}
x_{0} \\
x_{T}
\end{array}\right]
$$

where $N_{2}$ is defined in (11) and $\Phi_{1}:=P_{+}-F^{\top} P_{+} F, \Phi_{2}:=P_{+} W F^{\top}-F^{\top} P_{+} W$, and $\Phi_{3}:=$ $F^{\top} W\left(P_{+} W-I\right) F-W\left(P_{+} W-I\right)$.

Proof: First, consider Problem 2. From (4), (6) and (5), the optimal cost can be written as

$$
J_{2}^{o}=\int_{0}^{T} w^{\top}(t) L w(t) d t
$$

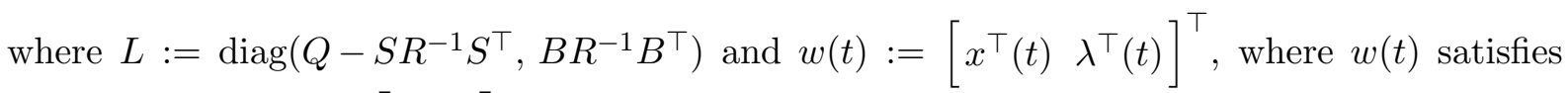
(9). Let now be $U:=\left[\begin{array}{ll}0 & I \\ I & 0\end{array}\right]$, A simple computation yields $H^{\top} U+U H=-2 L$, that can be replaced in the performance index. By using (5) we obtain

$$
\begin{aligned}
J_{2}^{o} & =-\frac{1}{2} \int_{0}^{T}\left[\dot{w}^{\top}(t) U w(t)+w^{\top}(t) U \dot{w}(t)\right] d t \\
& =-\frac{1}{2} \int_{0}^{T} \frac{d}{d t}\left(w^{\top}(t) w(t)\right) d t=x^{\top}(0) \lambda(0)-x^{\top}(T) \lambda(T) .
\end{aligned}
$$

By (9), one obtains exactly (14) with the corresponding values of $\Phi_{1}, \Phi_{2}$ and $\Phi_{3}$. Problem 1 can be treated by simply adding the terms $x^{\top}(T) P_{T} x(T)$ to $J_{2}^{o}$ by using (9).

\section{Concluding remarks}

A new approach has been presented for the solution of the finite-horizon LQ problem, in which the initial state is assigned, and the terminal state can both be assigned or simply weighted in the quadratic performance index. This method is based on the tools currently exploited to solve the infinite-horizon problems, i.e., the solution of an algebraic Riccati equation and of a Lyapunov equation, far easier to solve than a differential Riccati equation.

\section{References}


B.D.O. Anderson and J.B. Moore, 1989. Optimal Control: Linear Quadratic Methods. Prentice Hall International, London.

L.D. Berkovitz, 1974. Optimal Control Theory. Springer-Verlag, New York.

A. Ferrante, G. Marro, and L. Ntogramatzidis, 2003, Employing the algebraic Riccati equation for the solution of the finite-horizon LQ problem. In Proceedings of the 42nd IEEE Conference on Decision and Control (CDC 2003), Maui, Hawaii, USA, December.

H. KwakernaAk, And R. Sivan, 1972. Linear Optimal Control Systems, John Wiley \& Sons, New York.

V. Ionescu, C. OAră, And M. Weiss, 1999. Generalized Riccati theory and robust control, a Popov function approach (Wiley).

F.L. Lewis, And V. Syrmos, 1995. Optimal Control (John Wiley \& Sons, New York).

O.L. Mangasarian, 1966, Sufficient conditions for the optimal control of nonlinear systems. SIAM Journal on Control, 4(1):139-152.

B.P. Molinari, 1977. The time-invariant linear-quadratic optimal control problem. Automatica, 13:347-357.

H.L. Trentelman, A.A. Stoorvogel, and M. Hautus, 2001, Control theory for linear systems (Communications and Control Engineering. Springer, Great Britain).

K. Zhou, J. Doyle, And K. Glover, 1996. Robust and Optimal Control (Prentice Hall, New York). 